\title{
Compact THz Extended Interaction Oscillator Driven by a Pseudospark-sourced beam
}

\author{
Liang Zhang ${ }^{1}$, Jie Xie ${ }^{1,3}$, Wenlong $\mathrm{He}^{4}$, Kevin Ronald ${ }^{1}$, Alan D. R. Phelps ${ }^{1}$, Jin Zhang ${ }^{2}$, Xiaodong Chen ${ }^{2}$, \\ and Adrian W. Cross ${ }^{1}$ \\ ${ }^{1}$ Department of Physics, SUPA, University of Strathclyde, Glasgow G4 0NG, Scotland, UK \\ ${ }^{2}$ Department of Electronic Engineering, Queen Mary University of London, London, UK \\ ${ }^{3}$ School of Electronic Science and Engineering, University of Electronic Science \& Technology of China, Chengdu, China \\ ${ }^{3}$ College of Electronic Science and Technology, Shenzhen University, Shenzhen, 518060, China \\ e-mail: liang.zhang@strath.ac.uk
}

\begin{abstract}
The pseudospark discharge generated electron beam has the advantage of high current density and no need for an external guiding magnetic field. A sheet electron beam can be generated using a collimator with a sub-millimeter aperture. Simulation results showed that an extended interaction oscillator operating at $0.35 \mathrm{THz}$ could achieve an output power of 1.8 $\mathrm{kW}$. Simulations have been used to confirm that a $\pm 5 \mu \mathrm{m}$ tolerance will result in an acceptable performance.
\end{abstract}

\section{INTRODUCTION}

The generation of high-power radiation at sub-millimeter wavelengths in the terahertz frequency range is challenging, while high power, low cost, and compact sources at this frequency range have many applications. Conventional vacuum electron devices have the advantage of high-power capability compared with the solid-state sources. However, as the frequency increases, all the dimensions reduce, and their power level also drops quickly. One of the main limits is the current density from the thermionic cathode. The pseudospark (PS) discharge is a low-pressure gas discharge in an axially symmetric structure. It can produce a high-intensity electron beam pulse with beam current density up to $10^{4} \mathrm{~A} / \mathrm{cm}^{2}$ and brightness up to $10^{12} \mathrm{~A} /\left(\mathrm{m}^{2} \cdot \mathrm{rad}^{2}\right)$. Such a beam can propagate a certain distance (a few centimeters) due to the ion-channel generated during the discharge process and without the need of an external guiding magnet. Therefore, the PS discharge generated electron beam attracted great interest in driving a $\mathrm{THz}$ interaction circuit to generate high-power radiation. The PS discharge can only operate at pulse mode, a compact pulse power supply can be used to drive it, making it a low-cost portable source [1].

Different types of radiation sources driven by the PS discharge beam operating at different frequency bands have been experimentally studied. These include a Cherenkov maser at Ka-band, extended interaction oscillators (EIO) operating at W-band and G-band, and a backward wave oscillator (BWO) operating in G-band [2-7]. The EIO structure as an interaction region attracted significant interest due to its high beam-wave coupling impedance, which enables a shorter length beam-wave interaction region that can produce high output power. It is suitable for the PS beam as the EIO can tolerate a relatively large velocity spread and current loss which often occurs during the propagation of the PS beam. The EIO structure is a planar structure to make it suitable to be machined by wire electrode discharge machining.

Different shapes of the electron beam from the PS discharge can be achieved using different shapes of collimators. The EIOs operating at W-band driven by pencil beam and sheet beam were studied experimentally. The sheet beam EIO demonstrated much larger output power compared with the pencil beam. The reasons are the larger beam area in the sheet electron beam and the higher $\mathrm{R} / \mathrm{Q}$ of the planar interaction circuit.

In this paper, an EIO operating at $0.35 \mathrm{THz}$ was designed. A sheet electron beam was used to drive the interaction circuit. Particle-in-cell (PIC) simulations were used to investigate the performance.

\section{ELECTRON BEAM GENERATED FROM PS DISCHARGE}

The PS discharge is a low-pressure (typically in the range of 20-200 mTorr) gas discharge. A schematic of the PS discharge setup is shown in Fig. 1. Its structure consists of a hollow cathode with a central hole in the anode. The generated electron beam can be extracted through a central hole on the anode. The discharge voltage between cathode and anode is determined by the gas pressure and the gap distance. The uniformity of the electric field in the gap can be improved by multiple insulators.

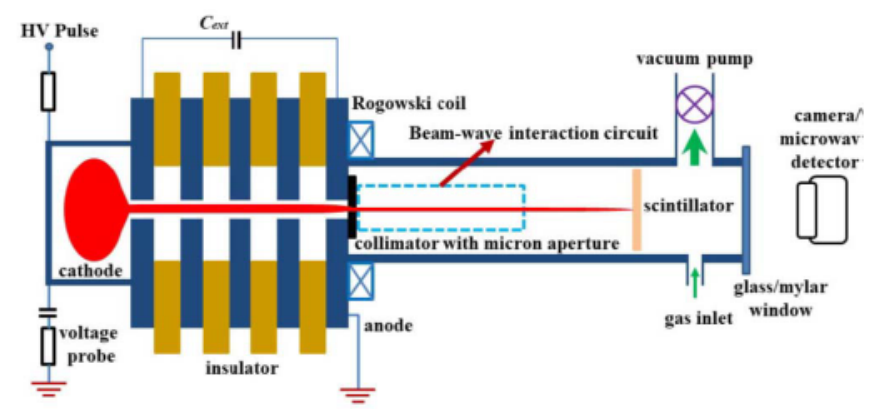

Figure 1. Schematic of PS discharge experiment.

The PS discharge process is commonly explained by three stages, including the pre-breakdown stage, the breakdown stage, 
as well as the super-dense discharging stage. properties of the PS generated electron beam including: (1) short pulse duration on the scale of tens nanosecond; (2) high-intensity electron beam with a density up to $10^{8} \mathrm{~A} / \mathrm{m}^{2}$ and brightness up to $10^{12}$ $\mathrm{A} /\left(\mathrm{m}^{2} \cdot \mathrm{rad}^{2}\right)$; (3) ion channel generation by the beam front. It creates unique self-focusing to the electron beam and no need for an external magnetic field $[8,9]$. The breakdown condition of the PS discharge is mainly determined by the dimensions of the cavity structure, the charging voltage, as well as the gas pressure. Fig. 2 shows typical waveforms of the discharge voltage and the beam current measured from the experiment. In the pre-breakdown stage, the beam current is small, and the charging voltage only drops slightly. The PS discharge occurs around $50-75 \mathrm{~ns}$, the beam voltage is about $50 \%-70 \%$ of the charging voltage, and the beam current is relatively large. At the super-dense discharge stage, the beam current reaches its maximum however the beam voltage is low.

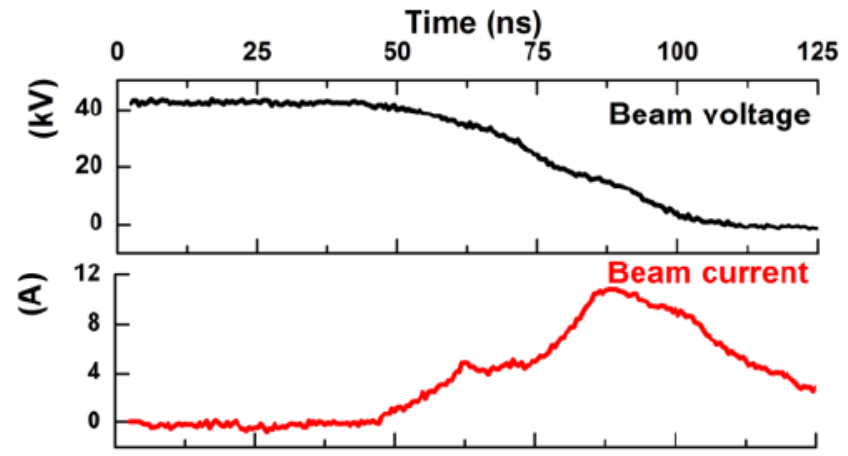

Figure 2. Typical curves of the discharge voltage and beam current of the PS experiment.

\section{DESIGN OF THE EIO INTERACTION CIRCUIT}

The EIO has the advantages of traveling-wave tubes and klystrons [10 -12]. A drawing of the interaction circuit consists of 11 resonant slots as shown in Fig. 3. The interaction circuit is strongly coupled with the symmetrical coupling cavities on both sides of the structure. From the beam-wave resonance conditions, the dimensions of the EIO can be estimated from the equations (1) to (3).

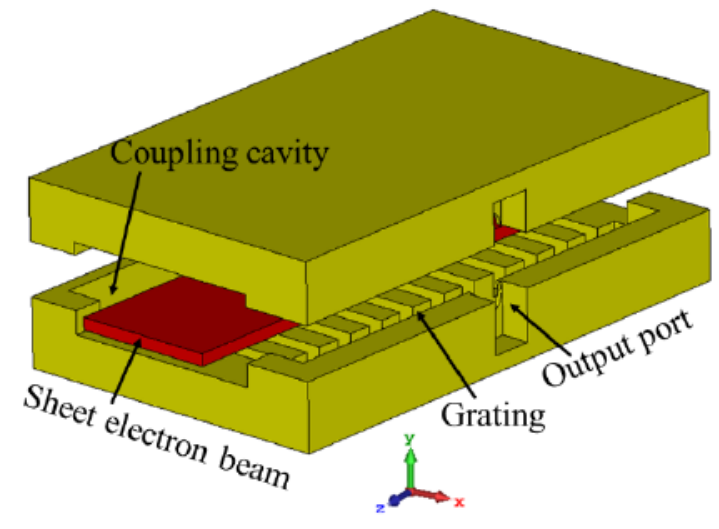

Figure 3. Schematic drawing of planar EIO interaction circuit.

$$
\begin{gathered}
\beta_{e} P=m \pi \\
\beta_{e}=2 \pi f / v_{e} \\
v_{e}=c \cdot \sqrt{1-\frac{1}{\left(1+\frac{U}{511}\right)^{2}}}
\end{gathered}
$$

Where $\beta_{e}, v_{e}, U, c$, and $f$ are the propagation constant of the electron beam, the beam velocity and voltage, the speed of light and the resonant frequency, respectively. $P$ is the period length of the multi-gap cavity. The $2 \pi$ mode, where $m=2$, is chosen as the operating mode to maximize the cavity impedance at the passband cut-offs. By given the operating frequency of the EIO ( $350 \mathrm{GHz})$, and the beam voltage ( $\sim 32 \mathrm{kV}$ to match with the PS discharge generated electron beam), its initial dimensions can be calculated from eq. 1- 3. Further optimizations on the dimensions were carried out by using the CST microwave studio to improve its quality factor $(\mathrm{Q})$ and $\mathrm{R} / \mathrm{Q}$. A higherquality factor helps to reduce the start-oscillation time, and a larger $\mathrm{R} / \mathrm{Q}$ value will improve the output power. The final dispersion of the EIO is shown in Fig. 4. The Detailed parameter sweep of the dimensions of the designed EIO can be found in paper [13]

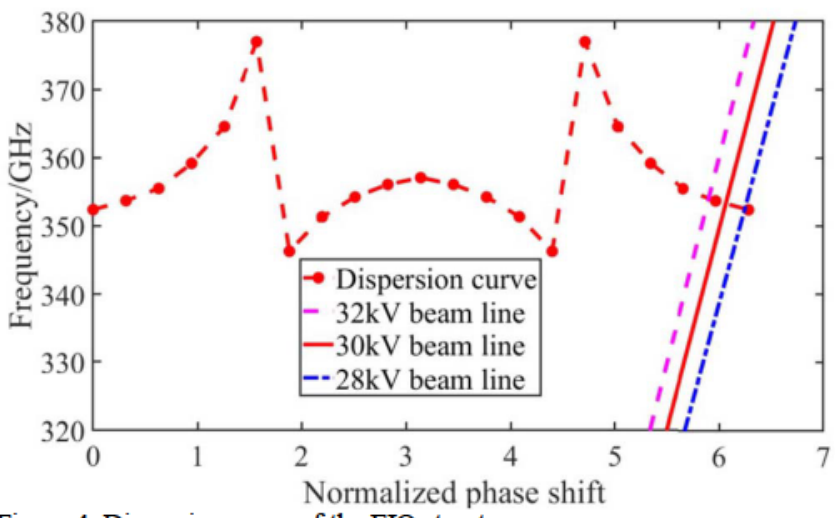

Figure 4. Dispersion curve of the EIO structure.

The performance of EIO was studied by particle-in-cell simulations and optimized by using CST particle studio. More details on the results of the parameter sweeps of the beam current, beam voltage and copper conductivity can be found in paper [13]. AT the optimal case, when driven by a beam voltage of $34 \mathrm{kV}$ and a beam current of $6 \mathrm{~A}$, which corresponds to a current density of $5.0 \times 10^{7} \mathrm{~A} / \mathrm{m}^{2}$, as well as considering a copper conductivity of $1.1 \times 10^{7} \mathrm{~S} / \mathrm{m}$, a stable output of $1.8 \mathrm{~kW}$ can be achieved, as shown in Fig. 5. The output frequency is $352 \mathrm{GHz}$, which is very close to the cold cavity resonance frequency of $352.4 \mathrm{GHz}$. The oscillation start-up time was about $4.3 \mathrm{~ns}$. The oscillation frequency stayed stable at around $352 \mathrm{GHz}$ when the beam voltage varies from 32 to $36 \mathrm{kV}$.

Machining tolerance is one of the challenges in the development of $\mathrm{THz}$ radiation sources. The further simulations showed with a $\pm 5 \mu \mathrm{m}$ tolerance, the maximum frequency shift remained within an acceptable value of $3.5 \mathrm{GHz}$ while being able to generate an output power of greater than $500 \mathrm{~W}$. 


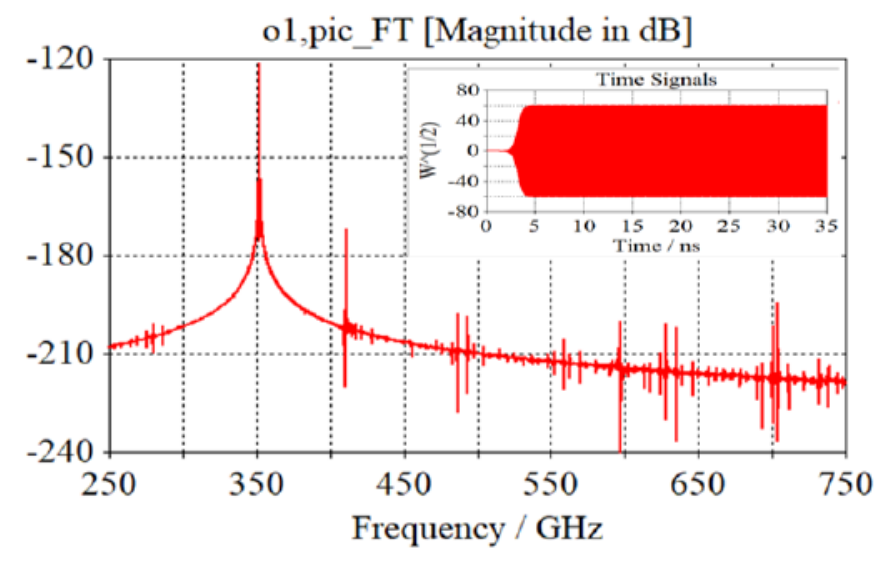

Figure 5. PIC simulation results of the EIO

\section{EXPERIMENTAL ARRANGEMENT}

The PS discharge experimental setup is shown in Fig. 6. A total external capacitor of $1.4 \mathrm{nF}$ was used and the charging voltage was fixed at $50 \mathrm{kV}$ to achieve the required beam voltage of $34 \mathrm{kV}$. A voltage pulse of $4 \mathrm{kV}$ was applied to a sharp pin at the center of the discharge cavity to trigger the discharge process. A stable repetition rate of $1000 \mathrm{~Hz}$ is expected to achieve.

The EIO structure is currently being manufactured using wire electrode discharge machining. Sub-mm wave measurements of its resonance frequency and quality factor will be carried out using a vector network analyzer, prior to installation and testing using the discharge experimental setup.

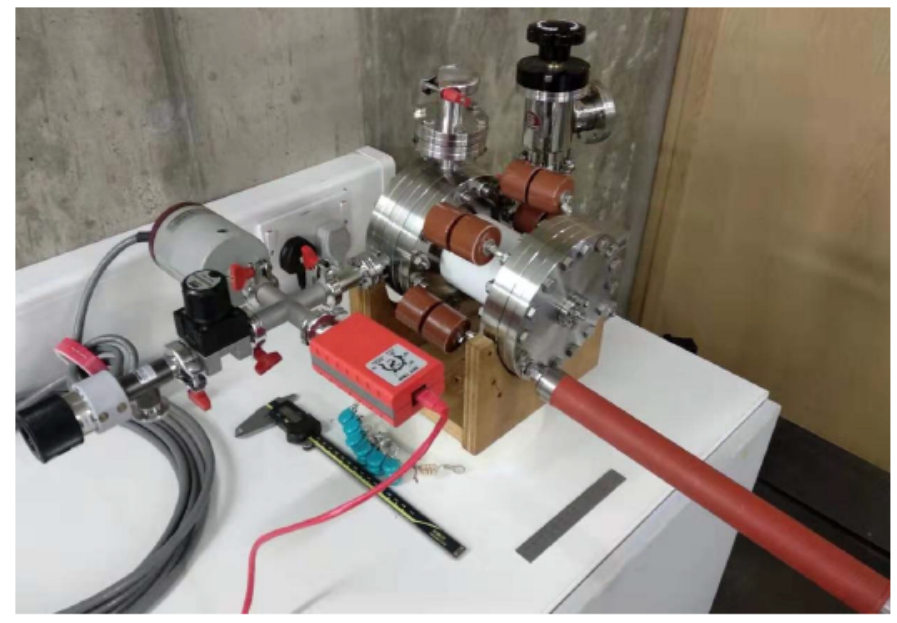

Figure 6. Experimental setup of the PS discharge chamber.

\section{SUMMARY}

This paper presents the design of a THz EIO driven by PS discharge generated electron beam which has the advantage of high current density and high brightness. The simulation shows an encouraging output of $1.8 \mathrm{~kW}$ at $0.35 \mathrm{THz}$ when it is driven by a beam voltage of $34 \mathrm{kV}$ and a beam current of $6 \mathrm{~A}$. The EIO structure is currently under machining and its experimental results will be presented later.

\section{ACKNOWLEDGMENT}

This work was supported by the U.K. Engineering and Physical Sciences Research Council (EPSRC) under Grant EP/S00968X/1 and Grant EP/S009582/1. Data underpinning this publication are openly available from the University of Strathclyde at: https://doi.org/10.15129/f66e9e87-c9d0-492aab0c-48cd6823belcs.

\section{REFERENCES}

[1] A. W. Cross, H. Yin, W. He, K. Ronald, A. D. R. Phelps, and L. C Pitchford, "Generation and application of pseudospark-sourced electron beams," Journal of Physics D-Applied Physics, vol. 40, no. 7, pp. 19531956, Apr 7 2007. DOI: 10.1088/0022-3727/40/7/018.

[2] $\mathrm{H}$. Yin et al., "Millimeter wave generation from a pseudosparksourced electron beam," Phys. Plasmas, vol. 16, no. 6, Jun. 2009, Art. no. 063105, doi: 10.1063/1.3155444.

[3] W. He et al., "Generation of broadband terahertz radiation using a backward wave oscillator and pseudospark-sourced electron beam," Applied Physics Letters, vol. 107, no. 13, Sep 28 2015. DOI: 10.1063/1.4932099.

[4] H. Yin et al., "Compact high-power millimetre wave sources driven by pseudospark-sourced electron beams," IET Microwaves, Antennas \&amp; Propagation, vol. 13, no. 11, pp. 1794 - 1798, Sep. 2019, doi: 10.1049/iet-map.2018.6190

[5] H. Yin, A. W. Cross, W. He, A. D. R. Phelps, and K. Ronald "Pseudospark experiments: Cherenkov interaction and electron beam post-acceleration," IEEE Transactions on Plasma Science, vol. 32, no. 1, pp. 233-239, Feb 2004. DOI: 10.1109/Tps.2004.823986

[6] H. Yin, A. W. Cross, A. D. R. Phelps, W. He, and K. Ronald, "Cherenkov interaction and post-acceleration experiments of high brightness electron beams from a pseudospark discharge," Nuclear Instruments \& Methods in Physics Research Section a-Accelerators Spectrometers Detectors and Associated Equipment, vol. 528, no. 1-2, pp. 378-381, Aug 1 2004. DOI: 10.1016/j.nima.2004.04.084.

[7] H. Yin, G. R. M. Robb, W. He, A. D. R. Phelps, A. W. Cross, and K. Ronald, "Pseudospark-based electron beam and Cherenkov maser experiments," Physics of Plasmas, vol. 7, no. 12, pp. 5195-5205, Dec 2000. DOI: Doi 10.1063/1.1319637.

[8] J. Zhao et al., "Experiments on W-band extended interaction oscillator with pseudospark sourced post-accelerated electron beam," Physics of Plasmas, vol. 24, no. 6, Jun 2017. DOI: 10.1063/1.4985684.

[9] H. Yin, A. W. Cross, A. D. R. Phelps, D. Zhu, W. He, and K. Ronald, "Propagation and post-acceleration of a pseudospark-sourced electron beam," Journal of Applied Physics, vol. 91, no. 8, pp. 5419-5422, Apr 15 2002. DOI: $10.1063 / 1.1459757$.

[10] Y. Yin, W. L. He, L. Zhang, H. B. Yin, C. W. Robertson, and A. W. Cross, "Simulation and Experiments of a W-Band Extended Interaction Oscillator Based on a Pseudospark-Sourced Electron Beam," IEEE Transactions on Electron Devices, vol. 63, no. 1, pp. 512-516, Jan 2016. DOI: $10.1109 /$ Ted.2015.2502950.

[11] G. X. Shu et al., "Demonstration of a Planar W-Band, kW-Level Extended Interaction Oscillator Based on a Pseudospark-Sourced Sheet Electron Beam," IEEE Electron Device Letters, vol. 39, no. 3, pp. 432435, Mar 2018. DOI: 10.1109/Led.2018.2794469.

[12] G. X. Shu et al., "Experimental demonstration of a terahertz extended interaction oscillator driven by a pseudospark-sourced sheet electron beam," Applied Physics Letters, vol. 112, no. 3, Jan 15 2018. DOI: 10.1063/1.5011102.

[13] J. Xie et al., "Study of a $0.35 \mathrm{THz}$ Extended Interaction Oscillator Driven by a Pseudospark-Sourced Sheet Electron Beam, " IEEE Transactions on Electron Devices, vol. 67, no. 2, pp. 652-658, Feb. 2020, doi: 10.1109/TED.2019.2957760. 